\title{
Corrigendum
}

Moosa Gabeleh*

\section{Corrigendum to the paper "Equivalence of the existence of best proximity points and best proximity pairs for cyclic and noncyclic nonexpansive mappings"}

https://doi.org/10.1515/dema-2021-0007

received February 18, 2021; accepted March 12, 2021

Abstract: The purpose of this short note is to present a correction of the proof of the main result given in the paper "Equivalence of the existence of best proximity points and best proximity pairs for cyclic and noncyclic nonexpansive mappings," Demonstr. Math. 53 (2020), 38-43.

Keywords: best proximity (point) pair, uniformly convex Banach space, noncyclic (cyclic) contraction

MSC 2020: 47H09, 46B20

\section{On the result in [1]}

The following theorem is the main result of [1].

Theorem 1.1. (Theorem 2.1 of [1]) Let $(A, B)$ be a nonempty, weakly compact and convex pair in a strictly convex Banach space $X$. Then every cyclic nonexpansive mapping defined on $A \cup B$ has a best proximity point if and only if every noncyclic nonexpansive mapping defined on $A \cup B$ has a best proximity pair.

We mention that the proof of Theorem 1.1 is based on the fact that the mapping $\mathcal{P}: A_{0} \cup B_{0} \rightarrow A_{0} \cup B_{0}$ is an isometry (see condition (2) of Proposition 1.8).

We mention that the isometricness of the mapping $\mathcal{P}$ means that both $\left.\mathcal{P}\right|_{A_{0}}$ and $\left.\mathcal{P}\right|_{B_{0}}$ are isometry, where in Proposition 1.8, we wrongly stated that $\left\|\mathcal{P}_{X}-\mathcal{P} y\right\|=\|x-y\|$ for all $(x, y) \in A_{0} \times B_{0}$.

To correct this, we need to introduce the following geometric notion.

Definition 1.2. Let $(A, B)$ be a nonempty pair of subsets of a metric space $(X, d)$ such that $A_{0}$ is nonempty. We say that the pair $(A, B)$ has the diagonal property provided that

$$
\left\{\begin{array}{l}
d\left(x_{1}, y_{1}\right)=\operatorname{dist}(A, B), \\
d\left(x_{2}, y_{2}\right)=\operatorname{dist}(A, B),
\end{array} \quad \Rightarrow d\left(x_{1}, y_{2}\right)=d\left(x_{2}, y_{1}\right),\right.
$$

for any $x_{1}, x_{2} \in A_{0}$ and $y_{1}, y_{2} \in B_{0}$.

For instance, if $(A, B)$ is a pair of nonempty subsets of a metric space $(X, d)$ such that $\operatorname{dist}(A, B)=0$, then $(A, B)$ has the diagonal property.

Also, every two parallel segments in the Euclidian plan $\mathbb{R}^{2}$ have the diagonal property. In more general, it can be shown that every nonempty, closed and convex pair in Hilbert spaces has the diagonal property.

\footnotetext{
* Corresponding author: Moosa Gabeleh, Department of Mathematics, Ayatollah Boroujerdi University, Boroujerd, Iran, e-mail: Gabeleh@abru.ac.ir, gab.moo@gmail.com
} 
We now have the following result regarding the projection mapping $\mathcal{P}$.

Proposition 1.3. Let $(A, B)$ be a nonempty, bounded, closed and convex pair in a reflexive and strictly convex Banach space $X$ such that $(A, B)$ has the diagonal property. Define $\mathcal{P}: A_{0} \cup B_{0} \rightarrow A_{0} \cup B_{0}$ as

$$
\mathcal{P}(x)= \begin{cases}\mathcal{P}_{A_{0}}(x) & \text { if } x \in B_{0}, \\ \mathcal{P}_{B_{0}}(x) & \text { if } x \in A_{0} .\end{cases}
$$

Then $\mathcal{P}$ is a relatively isometry, that is, $\|\mathcal{P} x-\mathcal{P} y\|=\|x-y\|$ for all $(x, y) \in A_{0} \times B_{0}$.

Proof. It follows from Proposition 1.8 of [1] that

$$
\|x-\mathcal{P} x\|=\|\mathcal{P} y-y\|=\operatorname{dist}(A, B), \quad \forall(x, y) \in A_{0} \times B_{0} .
$$

Since $(A, B)$ has the diagonal property, we obtain

$$
\|\mathcal{P} x-\mathcal{P} y\|=\|x-y\|,
$$

that is, $\mathcal{P}$ is a relatively isometry on $A_{0} \cup B_{0}$.

Thus, we need the condition diagonal property on the considered pair $(A, B)$ in Theorems 2.1 and 2.3 of [1].

Acknowledgements: This paper was written when Professor Hans-Peter A. Künzi was deceased. God bless him.

Conflict of interest: The author states no conflict of interest.

\section{Reference}

[1] M. Gabeleh and H. P. Künzi, Equivalence of the existence of best proximity points and best proximity pairs for cyclic and noncyclic nonexpansive mappings, Demonstr. Math. 53 (2020), 38-43. 